\title{
Assessing Gammapapillomavirus infections of mucosal epithelia with two broad- spectrum PCR protocols
}

\author{
Elisa M. Bolatti ${ }^{1,2 \dagger}$, Lea Hošnjak ${ }^{3 \dagger}$, Diego Chouhy ${ }^{1,2}$, Pablo E. Casal ${ }^{2}$, María F. Re-Louhau' ${ }^{1}$, Hebe Bottai ${ }^{4}$, \\ Kristina Fujs Komloš ${ }^{3}$, Mario Poljak ${ }^{3^{*}}$ and Adriana A. Giri ${ }^{1,2^{*}}$
}

\begin{abstract}
Background: Human papillomaviruses (HPVs) have been divided into mucosal and cutaneous types according to their primary epithelial tissue tropism. However, recent studies showed the presence of several cutaneous types in mucosal lesions and healthy mucosa from different anatomical sites.

Methods: Here, the HPV prevalence and type-specific distribution were assessed in a variety of mucosal samples from 435 individuals using a combination of two established broad-spectrum primer systems: Gamma-PV PCR and CUT PCR.

Results: Overall HPV prevalence in anal canal swabs, cervical cancer biopsies, genital warts and oral swabs was 85 , 47, 62 and 4\%, respectively. In anal canal swabs, Alpha-PVs were most frequently found (59\%), followed by Gamma(37\%) and Beta-PVs (4\%). The prevalence and persistence of HPV infection in the anal canal of 226 individuals were further explored. Overall HPV, Gamma-PVs and multiple HPV infections were significantly higher in men vs. women ( $p=0.034, p=0.027$ and $p=0.003$, respectively); multiple HPV infections were more common in individuals $\leq 40$ years $(p=0.05)$, and significantly higher prevalence of Gamma-PVs and multiple HPV infections was observed in HIV-1-positive vs. HIV-1-negative individuals ( $p=0.003$ and $p=0.04$, respectively). Out of 21 patients with follow-up anal swabs, only one persistent infection with the same type (HPV58) was detected.

Conclusions: Our findings suggest that Gamma-PVs (except species Gamma-6) are ubiquitous viruses with dual muco-cutaneous tissue tropism. Anal canal Gamma-PV infections may be associated with sexual behavior and the host immune status. This study expands the knowledge on Gamma-PVs' tissue tropism, providing valuable data on the characteristics of HPV infection in the anal canal.
\end{abstract}

Keywords: Gammapapillomavirus, Mucosal epithelia, Gamma-PV/CUT PCR assays, Anal canal, Prevalence, Persistence

\section{Background}

To date, more than 320 different human papillomavirus (HPV) types have been identified according to phylogenetic relationships of their complete L1 gene sequences, within five genera of the Papillomaviridae family (Alpha-, Beta-, Gamma-, Mu- and Nu-PV) [1-3]. Based on the epithelial tissue tropism, HPVs have been

\footnotetext{
* Correspondence: mario.poljak@mf.uni-lj.si; giri@ibr-conicet.gov.ar †Elisa M. Bolatti and Lea Hošnjak contributed equally to this work.

${ }^{3}$ Faculty of Medicine, Institute of Microbiology and Immunology, University of Ljubljana, Zaloška 4, 1000 Ljubljana, Slovenia

${ }^{1}$ Grupo Virología Humana, Instituto de Biología Molecular y Celular de Rosario (CONICET), Suipacha 590, 2000 Rosario, Argentina

Full list of author information is available at the end of the article
}

originally subdivided into mucosal and cutaneous types [1]. Mucosal HPV types, typically clustering to the Alpha-PV genus, which also contains several predominantly cutaneous HPV types, are associated with the development of pre-malignant and malignant lesions of the anogenital, oral and oropharyngeal epithelia [4]. AlphaPV infections of the anal canal have frequently been detected in human immunodeficiency virus (HIV-1)-infected men who have sex with men (MSM) $[5,6]$. Moreover, HIV-1-positive MSM have approximately 60 times higher risk of developing anal cancer than the general population $[7,8]$, and also show higher risk with respect to men who have sex with women (MSW) [9],

(c) The Author(s). 2020 Open Access This article is distributed under the terms of the Creative Commons Attribution 4.0 International License (http://creativecommons.org/licenses/by/4.0/), which permits unrestricted use, distribution, and 
women [10], and HIV-1-negative MSM [11], but in lower proportions.

Cutaneous HPVs are dispersed across all five HPV genera and have most frequently been detected in healthy skin samples, suggesting their commensal nature $[2,12]$. However, several studies showed a high prevalence of Beta- and Gamma-PVs at several anatomical sites, different from sites in which they were originally identified, including cutaneous and mucosal lesions and healthy mucosa, suggesting their double, mucocutaneous tissue tropism, and adding more questions about their clinical importance [13-15]. In contrast to Beta-PVs where the number of studies supporting their oncogenic potential in the development of skin cancer has increased over time [16], the role of Gamma-PVs in the development of malignant mucosal/cutaneous lesions has been poorly described [17-19]. Interestingly, our recent study, together with previous data, suggests a potential active role of Gamma-PVs in the development of premalignant skin lesions in immunocompetent individuals $[20,21]$. On the other hand, patients with a rare inherited immunodeficiency have been found to be uniquely susceptible to Gamma-PV-associated skin warts [22]. Furthermore, some Gamma-PV types, especially those belonging to the Gamma- 6 species, have been detected in Alpha-PV-negative anogenital warts $[23,24]$ and cervical precancerous lesions $[13,17,18,25,26]$.

Even though the presence of different HPV types has recently been demonstrated in the anal canal of men, the used methodologies only enabled the identification of a limited number of Alpha-, Beta- and/or GammaPV types [5, 10, 27, 28]. Moreover, very little is known about the natural history of infection with predominantly cutaneous HPVs in the anogenital region [27].

The objective of the present study was to assess the prevalence and type-specific distribution of a wide range of HPV types in a variety of mucosal samples, using a combination of two established broad-spectrum primer systems, enhancing the ability to detect Gamma-PVs. Additionally, the prevalence and persistence of HPV infection in the anal canal of HIV-1-positive and -negative men and women were comparatively evaluated.

\section{Methods}

\section{Patients' data, sample collection and processing}

Samples of total DNA $(n=458)$, extracted from 249 swabs of the anal canal (226 initial and 23 follow-up samples), 94 cervical cancer formalin-fixed paraffinembedded (FFPE) tissue samples, 21 genital warts (11 swabs and 10 biopsies) from different anatomical sites [foreskin $(n=4)$, scrotum $(n=2)$, penile glans $(n=2)$, perianal area $(n=4)$ and pubis $(n=9)]$, and 94 oral swabs collected during routine paternity testing, were obtained from the archival collection of samples of the
Instituto de Biología Molecular y Celular de Rosario (Rosario, Argentina) and the Institute of Microbiology and Immunology, Faculty of Medicine, University of Ljubljana (Ljubljana, Slovenia). Before subsequent analyses, all samples were collected, processed, and stored at $-80^{\circ} \mathrm{C}$, as described previously [11, 27, 29-32]. The adequacy of samples for downstream analyses was determined by PCR amplification of the human beta-globin gene, as previously described [33].

In more detail, and in order to compare the prevalence and persistence of HPV infection in the anal canal of HIV-positive and HIV-negative subjects, samples and data from 226 patients were mostly obtained from our previous studies on Alpha- and Beta-PV infections in the anal canal [11, 27]. Additionally, 23 follow-up samples from eligible individuals that were obtained after the conclusion of two previous studies were included in the present study. Subjects were 18 to 66 years old (median age $=33$ years $)$ and 20/226 (9\%) represented HIV-1 positive men. As all anal canal swab samples were collected during standard proctologic exams unfortunately information on the presence of specific anal lesions could not be obtained for all patients. For the 133 patients for whom clinical data were available, more than two-thirds $(90 / 133 ; 67.6 \%)$ had a clinically evident HPVrelated or HPV-unrelated anal pathology, including anal warts $(77 / 133 ; 57.9 \%)$, hemorrhoids $(5 / 133 ; 3.8 \%)$ and anal fissure $(3 / 133 ; 2.3 \%)$, as reported in our previous study [27], suggesting that anal warts were most probably the predominant HPV-related pathology in our patient population. Additionally, all male participants were MSM and had a history of receptive anal sexual intercourse. Unfortunately, no data on the sexual behavior of women could be obtained. Moreover, a total of 21 subjects (20 males and one female), age range of 22 to 42 years (median age $=30$ years), of which two men were HIV-1-positive, were enrolled in the current analysis of HPV persistence in the anal canal, with a follow-up anal swabs collected in period ranging from 2 to 82 months (average $=36$ months; median $=43$ months). At enrolment, 10/21 (47.6\%) of these patients had anal warts, $4 /$ $21(19.0 \%)$ had no clinically evident abnormalities, two patients had anal fissure and hemorrhoids, respectively, and no data were available for $5 / 21$ (23.8\%) patients (Table S2).

\section{Detection of HPV infection}

The presence of HPV infection was determined using two well-established HPV generic primer systems, Gamma-PV PCR [21] and CUT PCR [30]. All PCR reactions were performed as described previously [21], using the following reaction controls: a negative control $(5 \mathrm{ng}$ of human placental DNA) to check for the reaction's specificity, a reagent control $\left(\mathrm{H}_{2} \mathrm{O}\right.$ instead of the sample) 
to check for carry-over contamination, and 100 copies of cloned HPV4 or HPV10 in a background of 5 ng human placental DNA as a positive control per PCR run. All pre- and post-PCR procedures were carried out in separate cabinets and rooms. Amplicons derived from CUT $(\approx 370 \mathrm{bp})$ and Gamma-PV PCR systems ( $\approx 158 \mathrm{bp})$ were sequenced by Sanger methodology. The obtained nucleotide sequences were compared to available HPVsequences in the GenBank database (https://www.ncbi. nlm.nih.gov/genbank/), using the Blast algorithm (https://blast.ncbi.nlm.nih.gov/Blast.cgi). Following CUT PCR, a novel putative HPV type was determined when the fragment sequence showed less than $90 \%$ nucleotide identity to L1 ORFs of any of the previously known HPV types [1]. The same criterion was applied to classify types/putative types derived from the Gamma-PV PCR, which is based on the amplification of partial HPV E1 sequences, due to the similar nucleotide identities obtained in a pairwise comparison analysis between L1 and E1 ORFs, as shown previously [21].

Additionally, in the analysis of HPV persistence in the anal canal, the obtained results were compared to the results of HPV typing using the commercially available Linear Array HPV Genotyping Test (Roche Diagnostics, Mannheim, Germany) and RHA Kit Skin (Beta) HPV assay (RHA; Diassay BV, Rijswijk, The Netherlands), as described previously $[11,27]$.

\section{Phylogenetic analysis of novel putative HPV types}

Sequences of E1 gene regions of 166 representative HPV types from the Gamma-PV genus, available at http:// www.nordicehealth.se/hpvcenter/ and Papillomavirus episteme (http://pave.niaid.nih.gov), were used as a database for the phylogenetic analysis of novel putative HPV types. Multiple sequence and pairwise alignments were constructed using the ClustalW algorithm of the MEGA6 software package [34] at the amino acid (aa) level. The alignment of novel putative HPV E1 sequences was obtained with MAFFT's "Align fragment sequences to an MSA" tool [35]. The phylogenetic relationships between representative Gamma-PV types and novel putative HPV E1 sequences were inferred by Bayesian analysis using Beast version 1.7.5 [36]. To do so, Markov Chain Monte Carlo (MCMC) simulations were performed during $2 \times 10^{7}$ generations, sampling one state every 1000 generations, with a burnin of $10 \%$. The setting "Create tree log file with branch length in substitutions" was selected to obtain the phylogram log file. The evolutionary substitution model selected for each run was $G T R+I+\Gamma$. Statistical convergence of MCMC was assessed visually by the traceplot and by calculating the effective sample size using TRACER v1.4 (available at http://beast.bio.ed.ac.uk/Tracer). The maximum clade credibility tree across all the plausible trees generated by BEAST was then computed with the TreeAnnotator program available in the BEAST package.

\section{Statistical analysis}

The statistical analysis of categorical variables was performed by Chi square and Fisher Exact tests. Multivariate analysis (logistic regression) was performed for all variables. $p$ values below 0.05 were regarded as statistically significant.

\section{Nucleotide sequence accession number}

The GenBank/EMBL/DDBJ accession numbers for the novel HPV putative types reported in this paper are: EP26 (MK510728), EP27 (MK510729), EP28 (MK510730), EP29 (MK510731).

\section{Results}

\section{HPV detection and typing using Gamma-PV and CUT PCR} systems

A total of 71 different HPV types/putative types (27 Alpha-, 7 Beta- and 37 Gamma-PV), clustering to 30 PV species (9 Alpha-, 3 Beta- and 18 Gamma-PV), were identified in HPV-positive mucosal samples using both broad-spectrum PCR protocols (Table 1, Table S1). It should be noted that only five HPV types (HPV38, HPV133, HPV161, HPV135 and HPV180) were consistently simultaneously detected in the same samples by both primer systems. In addition, four novel putative HPV types (EP26, EP27, EP28, EP29) were identified in this set of samples, all of them being found in anal canal swabs with the Gamma-PV PCR primer system (Table 1, Table S1). Phylogenetic relationships between these novel putative HPV types and sequences of E1 gene regions of 166 representative HPV types from the Gamma-PV genus are shown in Fig. 1. Putative HPV type EP26 clusters within the species Gamma-20 and shares a 90\% E1 ORF nucleotide identity with HPV163. EP27 belongs to the species Gamma-9 and shows the highest nucleotide identity with HPV216 (88\%). On the other hand, EP28 exhibits an $87 \%$ nucleotide identity with HPV-mSE379, clustering within the Gamma-8 species, while EP29 belongs to the Gamma-10 species, sharing a 90\% nucleotide identity with HPV180.

\section{HPV prevalence and genera distribution in the mucosal epithelia}

Overall, HPV DNA was present in 55\% (253/458) of all tested mucosal samples and HPV prevalence was determined at 58\% (253/435) (Table 1). Specifically, HPV prevalence in anal canal swabs, cervical cancer biopsies, genital warts and oral swabs was estimated at 85\% (192/ 226), 47\% (44/94), 62\% (13/21) and 4\% (4/94), respectively. Additionally, 25\% (116/458) of included samples 
Table 1 HPV detection in 458 samples of mucosal epithelia

\begin{tabular}{|c|c|c|c|c|c|c|c|}
\hline & $\begin{array}{l}\text { Patients } \\
(n)\end{array}$ & $\begin{array}{l}\text { Samples } \\
(n)\end{array}$ & $\begin{array}{l}\text { HPV-prevalence } \\
n(\%)\end{array}$ & $\begin{array}{l}\text { No. of detected } \\
\text { HPV types }\end{array}$ & $\begin{array}{l}\text { No. of detected } \\
\text { novel putative } \\
\text { HPV types }\end{array}$ & $\begin{array}{l}\text { No. of detected } \\
\text { HPV species }\end{array}$ & $\begin{array}{l}\text { Detected multiple } \\
\text { infections } \\
n(\%)\end{array}$ \\
\hline Anal canal swabs & 226 & 249 & $192(85)$ & 67 & 4 & 29 & $105(48)$ \\
\hline Cervical cancer biopsies & 94 & 94 & $44(47)$ & 6 & 0 & 3 & $7(7)$ \\
\hline Genital wart samples & 21 & 21 & $13(62)$ & 3 & 0 & 3 & $1(5)$ \\
\hline Oral swabs & 94 & 94 & $4(4)$ & 3 & 0 & 3 & $3(3)$ \\
\hline Total & 435 & 458 & $253(55)$ & $70^{a}$ & 4 & $30^{\mathrm{b}}$ & $116(25)$ \\
\hline
\end{tabular}


HPV58 (anal canal and cervical cancer); HPV18 (anal canal, cervical cancer and oral swabs); HPV38 (anal canal and oral swabs)

${ }^{\mathrm{b}}$ The following PV species were identified in different sets of samples: Alpha-7 (anal canal, cervical cancer and oral swabs); Alpha-9 (anal canal and cervical cancer); Alpha-10 and Gamma-18 (anal canal and genital warts); Beta-2 (anal canal and oral swabs)

contained multiple HPV infections, most frequently in swabs of the anal canal (105/226; 48\%) (Table 1).

As shown in Fig. 2 and Table S1, in swabs of the anal canal members of the Alpha-PV genus were the most frequently detected (113/192; 59\%), followed by Gamma- (71/192; 37\%) and Beta-PV genera (8/192; 4\%). Alpha-PV genus types were also the most frequently detected in cervical cancer biopsies $(43 / 44 ; 98 \%)$ and genital wart samples $(13 / 14 ; 93 \%)$. While HPV types clustering to the species Alpha-10 were the most frequent in anal canal swabs $(50 / 192 ; 26 \%)$ and genital wart samples $(11 / 14 ; 79 \%)$, HPVs grouped within the species Alpha-9 were most frequently identified in cervical cancer biopsies (36/44; 82\%). The prevailing HPV type detected in both anal canal swabs $(37 / 192 ; 19 \%)$ and genital wart samples $(7 / 14 ; 50 \%)$ was HPV6 (Alpha-10) and, as expected, HPV16 (Alpha-9) was the most prevalent in cervical cancer biopsies $(32 / 44 ; 73 \%)$. Nevertheless that a wide variety of Gamma-PVs, clustering to different Gamma-PV species, were detected in the anal canal (Fig. 2, Table S1), those grouped within the species Gamma-6 were the most frequently identified (11/192; $6 \%)$. It should be noted that unclear electropherograms from which the prevailing HPV type/s and species could not be determined (Gamma-X) were obtained from 18 Gamma-PV-positive anal canal swab samples (Fig. 2, Table S1). The frequency of HPV infection in oral swab samples was relatively low and the majority of positive samples contained Alpha-PVs [HPV10 (Alpha-2) and HPV18 (Alpha-7)] or Beta-PVs [HPV38 (Beta-2)] (Fig. 2, Table S1).

\section{HPV prevalence and persistence in samples of the anal canal}

The overall prevalence of HPV infection in the anal canal of the 226 patients included in the study was estimated at 79\% (Table 2). Particularly, Alpha-, Beta- and Gamma-PVs were present in 59, 3 and 35\% of the initial samples obtained from all study participants at the first visit, respectively. In addition, multiple infections were detected in $43 \%$ of included samples.

In comparison to women, the prevalence of overall HPV, Gamma-PV and multiple HPV infections was significantly higher in anal canal swab samples of men ( $82 \%$ vs. $69 \%, p=0.034 ; 38 \%$ vs. $22 \%, p=0.027$ and $49 \%$ vs. $26 \%, p=0.003$, respectively) (Table 2). Multiple HPV infections were slightly more common among 18-30 and 31-40-year-old patients (45 and 49\%, respectively), in comparison to individuals older than 40 years $(27 \%)$ ( $p=$ 0.05). A significantly higher prevalence of Gamma-PVs and multiple HPV infections was observed in HIV-1positive vs. HIV-1-negative individuals (65\% vs. $32 \%, p=$ 0.003 and $65 \%$ vs. $41 \%, p=0.04$, respectively). All differences between gender, age and HIV-1 status observed during univariate analyses were additionally confirmed using multivariate analyses (Table 3). Thus, men had a higher risk for overall HPV infections, Gamma-PV infections and multiple HPV infections than women, and HIV-1-positive subjects had a higher risk for infections with Gamma-PV and for multiple HPV infections than HIV-1-negative individuals.

As shown in Table S2, out of 21 patients with followup anal swabs, persistent infection with the same HPV type was detected only in a single patient (HPV58, patient No.18; Table S2). Additionally, two individuals were HPV-negative throughout the follow-up period (patient No. 8 and No. 21; Table S2). A total of 52\% (11/ 21) individuals were infected with different HPV types/ putative types in the initial and follow-up anal samples, and 29\% (6/21) had HPV-positive initial samples but tested negative in the follow-up samples. Only one patient tested HPV-positive in follow-up sample, while being previously HPV-negative (patient No. 2; Table S2).

\section{Discussion}

With the exception of Alpha-PVs, which are etiologically associated with the development of more than 99\% of cases of cervical cancer, $70-90 \%$ of cases of anal and vaginal cancers, $40 \%$ of cases of vulvar cancer, $47 \%$ of cases 


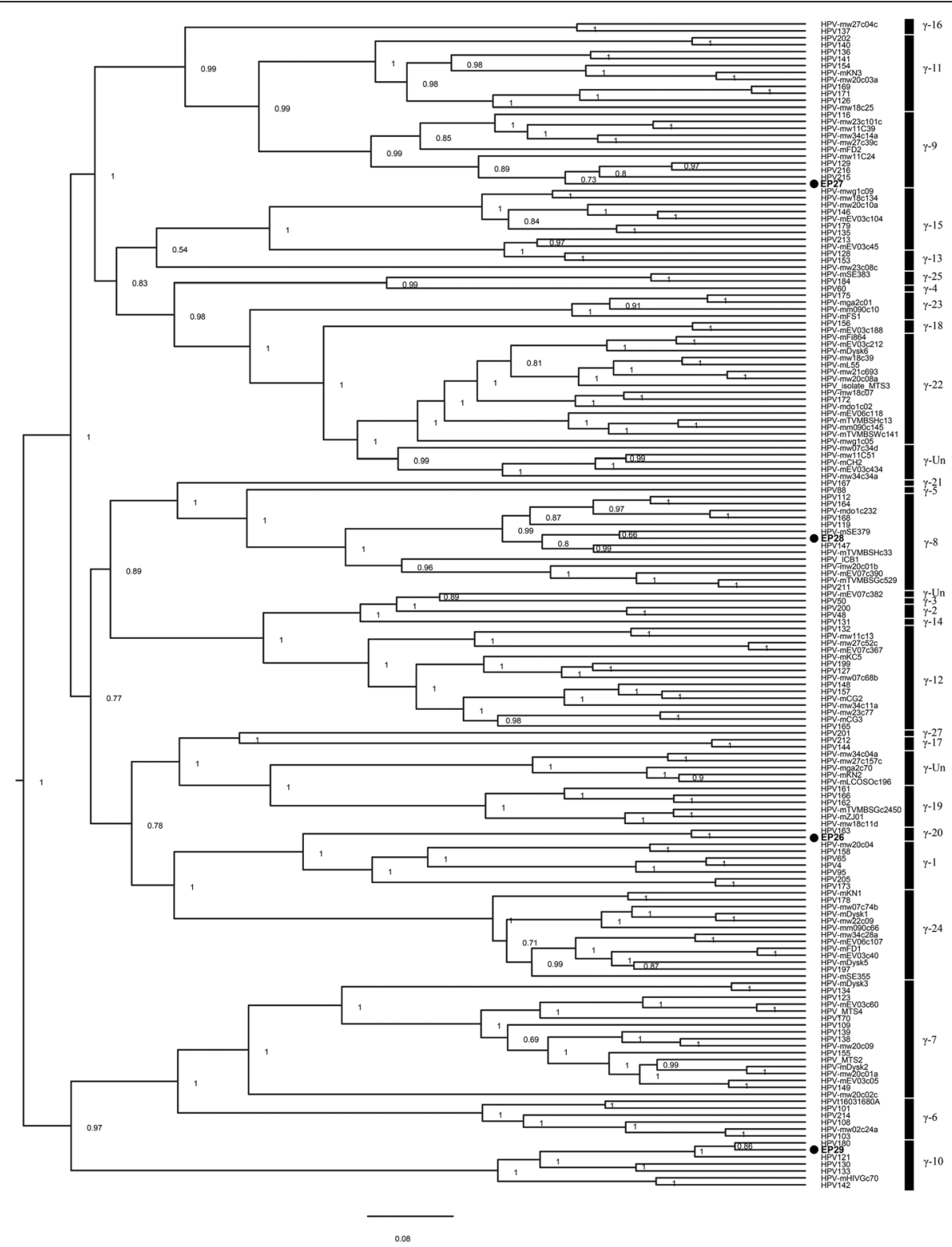

Fig. 1 Phylogenetic tree of novel putative HPV types and 166 representative HPV sequences from Gamma-PV genus. Phylogenetic analysis of partial E1 nucleotide sequences obtained with Gamma-PV PCR ( $158 \mathrm{bp}$ ) and the phylogenetic position of four novel putative HPV types (EP26, EP27, EP28, EP29) identified in this study. Only bayesian posterior probability (BPP) values of $>0.50$ are shown. Novel putative HPV types identified in this work are indicated with solid dots. Some clades were collapsed to facilitate the visual analysis. The raw phylogenetic trees are available upon request

of penile cancer, $25-30 \%$ of cases of oropharyngeal cancer, and more than $90 \%$ of cases of genital warts and laryngeal papillomas [37, 38], knowledge is limited concerning prevalence and clinical importance of other HPV genera in the mucosal epithelia. The present analysis of 458 mucosal samples, including biopsies of genital warts and cervical cancer as well as anal and oral swabs, enabled the identification of a large number of HPV types, clustering to a diverse range of species of Alpha-, Beta- and Gamma-PV genera.
Interestingly, only five HPV types were simultaneously detected by Gamma-PV and CUT PCR assays, showing the differential capacities of both primer systems on detecting diverse HPV types, as indicated previously [21]. Nevertheless that one set of broadspectrum primers targeting the E1 gene has been described previously [39], the mentioned CP primers can mostly detect Alpha- and Beta-PV types and have different specificities than those used in our Gamma-PV assay (Table S3). 


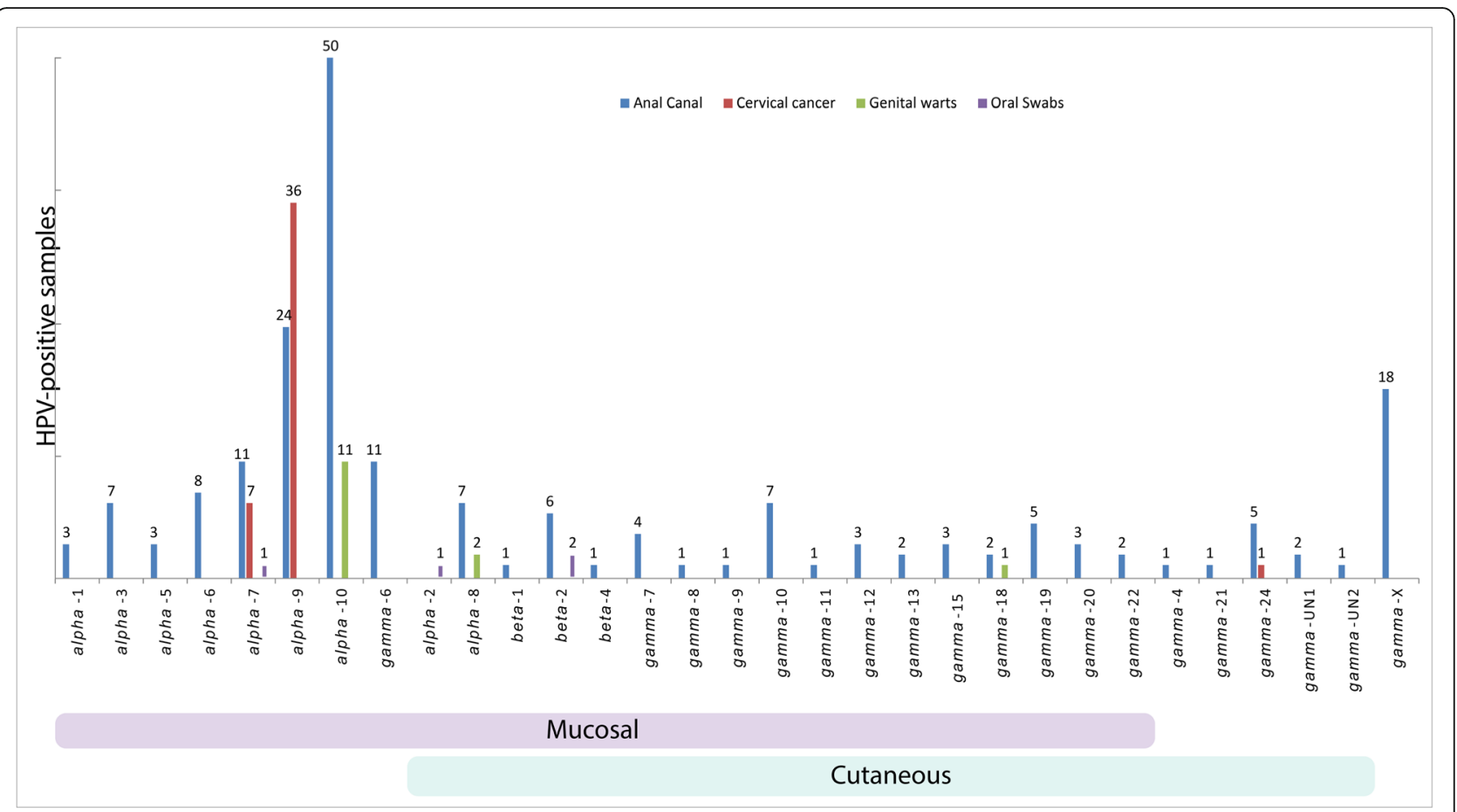

Fig. 2 Detection of Alpha-, Beta- and Gamma-PVs in 458 samples of mucosal epithelia. Color bars under X axis show HPV species according to cutaneous and mucosal tissue tropism [1]. HPV species over merged bars exhibit dual tissue tropism. Gamma-X refers to sequences grouped in the genus Gamma-PV from which the prevailing HPV types/s and species could not be determined

Thirty-six different Gamma-PVs, clustering into 18 species, with two of them described for the first time and not yet officially recognized (Gamma-Un1, GammaUn2; Fig. 2 and Table S1), were identified in the present study. Among all Gamma-PV-positive samples, members of the Gamma- 6 species were the most frequently detected $(11 / 73 ; 15 \%)$. Interestingly, none of them were identified in a variety of 653 cutaneous samples with the same testing approach [21]. These results, together with previously published findings $[13,17,26,28,40-43]$, suggest the possible adaptation of the mentioned HPV types to the mucosal epithelium [44].

Our observations are not surprising since previous studies have demonstrated that typically cutaneous HPV types (Beta- and Gamma-PVs) were relatively common in the mucosal epithelia, suggesting a possible dual tissue tropism of the majority of HPV species [1, 13, 32, 28, 45, 46]. In addition, two Gamma-PV types were identified in a

Table 2 Prevalence of overall HPV, Alpha-, Beta- and Gamma-PV infections in the anal canal of 226 individuals

\begin{tabular}{|c|c|c|c|c|c|c|c|c|c|c|c|}
\hline Variable & No. of patients & $\begin{array}{l}\text { All HPVs } \\
n(\%)\end{array}$ & $p$ & $\begin{array}{l}\text { Alpha-PVs } \\
n(\%)\end{array}$ & $p$ & $\begin{array}{l}\text { Beta-PVs } \\
n(\%)\end{array}$ & $p$ & $\begin{array}{l}\text { Gamma-PVs } \\
n(\%)\end{array}$ & $p$ & $\begin{array}{l}\text { Multiple infections } \\
n(\%)\end{array}$ & $p$ \\
\hline All Subjects & 226 & $179(79)$ & & $133(59)$ & & $7(3)$ & & $78(35)$ & & $98(43)$ & \\
\hline \multicolumn{12}{|l|}{ Gender } \\
\hline Female & 51 & $35(69)$ & 0.034 & $28(55)$ & 0.515 & $1(2)$ & 0.59 & $11(22)$ & 0.027 & $13(26)$ & 0.003 \\
\hline Male (MSM) & 175 & $144(82)$ & & $105(60)$ & & $6(3)$ & & $67(38)$ & & $85(49)$ & \\
\hline \multicolumn{12}{|l|}{ Age } \\
\hline $18-30$ years & 97 & $76(78)$ & 0.69 & $59(61)$ & 0.86 & $4(4)$ & 0.40 & $32(33)$ & 0.17 & $44(45)$ & \\
\hline $31-40$ years & 88 & $72(82)$ & & $50(56)$ & & $1(1)$ & & $36(40)$ & & $43(49)$ & 0.05 \\
\hline 41-66 years & 41 & $31(76)$ & & $24(59)$ & & $2(5)$ & & $10(24)$ & & $11(27)$ & \\
\hline \multicolumn{12}{|l|}{ HIV-1 status } \\
\hline HIV-1-positive & 20 & $18(90)$ & 0.21 & $14(70)$ & 0.29 & $2(7)$ & 0.103 & $13(65)$ & 0.003 & $13(65)$ & 0.04 \\
\hline HIV-1-negative & 206 & $161(78)$ & & $119(58)$ & & $5(3)$ & & $65(32)$ & & $85(41)$ & \\
\hline
\end{tabular}


Table 3 Multivariate Analysis of variables associated with HPV infection in the anal canal of 226 individuals

\begin{tabular}{|c|c|c|c|c|c|c|c|c|c|}
\hline \multirow[t]{2}{*}{ Variable } & \multicolumn{3}{|c|}{ HPV infection } & \multicolumn{3}{|c|}{ Gamma-PV infection } & \multicolumn{3}{|c|}{ Multiple HPV infection } \\
\hline & $n(\%)$ & $p$ & $\begin{array}{l}\text { Odds Ratio } \\
\text { [95\% Cl] }\end{array}$ & $n(\%)$ & $p$ & $\begin{array}{l}\text { Odds Ratio } \\
\text { [95\% Cl] }\end{array}$ & $n(\%)$ & $p$ & $\begin{array}{l}\text { Odds Ratio } \\
\text { [95\% Cl] }\end{array}$ \\
\hline \multicolumn{10}{|l|}{ Gender } \\
\hline Female & $35(69)$ & 0.0369 & $2.12[1.05-4.31]$ & $11(22)$ & 0.048 & 2.25 [1.08-4.69] & $13(26)$ & 0.023 & $2.29[1.12-4.67]$ \\
\hline Male (MSM) & $144(82)$ & & & $67(38)$ & & & $85(49)$ & & \\
\hline \multicolumn{10}{|l|}{ HIV status } \\
\hline HIV-1-positive & $18(90)$ & NS & - & $13(65)$ & 0.0124 & $3.47[1.31-9.22]$ & $13(65)$ & 0.049 & 2.38 [1.01-6.37] \\
\hline HIV-1-negative & $161(78)$ & & & $65(32)$ & & & $85(41)$ & & \\
\hline
\end{tabular}

NS not significant

surface swab of a genital wart and a cervical cancer biopsy sample, possible as part of the normal mucosal microbiota, as reported previously $[23,26]$.

Although using CUT primers several high- and lowrisk Alpha-PV types were identified in cervical cancer biopsies, a significantly lower HPV prevalence was found in the present study in comparison with previously published data [4]. The mentioned discrepancy could be a result of the fact that CUT primers were designed to detect the so-called "cutaneous" HPV types that are distributed across all five HPV genera [30]. Although CUT primers are able to detect mucosal types, their sensitivity for detection of Alpha-PV is much lower in comparison to other approaches using well-known Alpha-PV PCR primers, such as MY09/11, GP5+/6+ or SPF10, as previously reported [47]. Therefore, the CUT primers are not recommended for detection of clinically most relevant mucosal Alpha-PV HPV types associated with the development of several anogenital neoplasms, including cervical cancer. Instead, CUT primers can be considered as an additional tool for epidemiological studies, in combination with other testing approaches, such as the Gamma-PV PCR assay and/or with standard Alpha-PV primers, to explore the presence and HPV type diversity. Additionally, it should be considered that amplification of DNA sequences that are approximately $370 \mathrm{bp}$ long could be compromised in FFPE tissue samples, leading to an underestimation of HPV prevalence.

In line with previous epidemiological studies that have analyzed the prevalence of HPV infection in anal canal of MSM [5, 27, 28], MSW [10] and women [9], in the present study significantly higher overall HPV prevalence and rate of multiple HPV infections were detected in swabs of the anal canal of MSM in comparison to women ( $p=0.034$ and $p=0.003$, respectively). Nevertheless that the prevalence of Alpha-PV infections was similar in anal canal of MSM and women $(p=0.515)$, interestingly, the prevalence of Gamma-PVs was significantly higher among MSM $(p=0.027)$. Although the mentioned differences may have originated from the use of different HPV detection methods in different studies, it is likely that receptive anal sexual intercourse might indeed have resulted from more frequent acquisition of Gamma-PVs, as suggested previously for Alpha- and Beta-PVs [27, 48, 49]. On the other hand, it should be considered that anal HPV infections, especially with Beta- and Gamma-PVs, may occur through self- or partner- inoculation [50, 51].

In line with previous observations that younger age could be associated with the higher risk for acquiring anal HPV infection in men $[10,52]$, in the present study significantly higher prevalence of multiple HPV infections was detected in subjects that were 18-30 and 3140 years old at the time of the study in comparison to older individuals (41-66 years) $(p=0.05)$. The agespecific HPV infection prevalence trends in the anogenital region may have resulted from younger subjects being more sexually active than older individuals, with up to 3-fold higher number of sexual partners [52].

Since Beta- and Gamma-PV prevalence data among HIV-1-positive individuals are conflicting [5, 27, 28, 41], HPV prevalence according to patients' HIV-1 infection status was further investigated in the present study. In concordance with former reports $[28,41]$, the prevalence of Gamma-PVs and multiple HPV infections was significantly higher in HIV-1-positive subjects in comparison to HIV-1-negative individuals $(p=0.003$ and $p=0.04$, respectively). As the contradictory results obtained in epidemiological studies could be attributed to differences in the immune status of HIV-1-infected individuals and to the heterogeneity of enrolled patients and HPV detection methods used, additional studies are warranted to further explore the association(s) between HIV-1 and anal HPV infection.

Due to the scarce knowledge on the natural history of non-Alpha-PV infection in the anal canal [27], 21 patients were prospectively followed-up in our study. While all Beta- and Gamma-PV infections were found to be transient, a persistent Alpha-PV infection was detected in one patient. Although transient Gamma-PV infections have previously been described in healthy skin samples [53], in studies using reverse-line blot 
hybridization techniques, it has been demonstrated that Alpha- and Beta-PVs can establish persistent infections of the anal canal [11, 27]. It should be noted that in the present study, HPV infections were detected using broadspectrum primers, enhancing the ability to amplify Gamma-PVs, followed by direct sequencing of PCR products, which may represent a limitation, as it could have led to the underestimation of some HPVs causing persistent infections and being present in lower viral loads. Therefore, it is possible that Gamma-PVs also cause transient infections of the anal canal, mostly having a commensal role and being transmitted through sexual and non-sexual routes.

\section{Conclusions}

Based on results of our study and findings published previously, it could be concluded that Gamma-PVs are ubiquitous viruses with a wide tissue tropism, as they were detected in both mucosal and cutaneous sites, with the exception of members of the Gamma-6 species, which most probably only colonize mucosal ecological niches $[21,30,53]$. Our results provide new evidence that Gamma-PV infections of the anal canal may be associated with sexual behavior and the immune status of infected individuals. In conclusion, the present study expands the knowledge on Gamma-PVs' tissue tropism, providing valuable data on the characteristics of HPV infection in the anal canal.

\section{Supplementary information}

Supplementary information accompanies this paper at https://doi.org/10. 1186/s12879-020-4893-3.

Additional file 1: Table S1. HPV types/putative types detected by Gamma-PV and CUT PCR assays. Novel putative HPV types are depicted in bold. Un: species not included in the current HPV taxonomy. *: The same HPV/putative type was found in different sets of samples. Gamma$X$ refers to sequences grouped in the genus Gamma-PV from which the prevailing HPV type/s and species could not be determined.

Additional file 2: Table S2. Distribution of HPV types in 21 initial and 23 follow-up anal canal swab samples from 21 individuals. Linear Array HPV Genotyping Test (Roche Diagnostics GmbH, Mannheim, Germany) is based on the reverse-line blot hybridization technique to detect the following 37 different mucosal HPV types distributed in 11 Alpha-PV species (high-risk HPV types depicted in bold): Alpha-1 (HPV42), Alpha-3 (HPV61, HPV62, HPV72, HPV81, HPV83, HPV84, CP6108), Alpha-5 (HPV26, HPV51, HPV69, HPV82, IS39), Alpha-6 (HPV53, HPV56, HPV66), Alpha-7 (HPV18, HPV39, HPV45, HPV59, HPV68, HPV70), Alpha-8 (HPV40), Alpha-9 (HPV16, HPV31, HPV33, HPV35, HPV52, HPV58, HPV67), Alpha-10 (HPV6, HPV11, HPV55), Alpha-11 (HPV73, HPV64), Alpha-13 (HPV54), Alpha-14 (HPV71). RHA Kit Skin (Beta) HPV assay (RHA; Diassay BV, Rijswijk, The Netherlands) detects the following 25 different Beta-PV types by reverse-line blot hybridization technique: Beta-1 (HPV5, HPV8, HPV12, HPV14, HPV19, HPV20, HPV21, HPV24, HPV25, HPV36, HPV47, HPV93), Beta-2 (HPV9, HPV15, HPV17, HPV22, HPV23, HPV37, HPV38, HPV80), Beta-3 (HPV49, HPV75, HPV76), Beta-4 (HPV92), Beta-5 (HPV96). HPV types (or negative results) identified simultaneously by more than one HPV test are depicted in bold. Samples containing possible unknown HPV type(s) are indicated as HPV-X. Pos: Positive; Neg: Negative; N/A: Not Analyzed.
Additional file 3: Table S3. Alignments of the forward and reverse primer sequences of CUT PCR, Gamma-PV PCR and CP PCR with corresponding regions of L1 and E1 ORFs of 64 selected Gamma-PV types. Lines and characters represent identical and mismatched nucleotides, respectively. Conserved sequences at the $3^{\prime}$ regions of Gamma-PV primers are highlighted in grey. Degenerate nucleotides of primers: $\mathrm{i}=$ inosine, $\mathrm{y}=\mathrm{c} / \mathrm{t}, \mathrm{r}=\mathrm{a} / \mathrm{g}, \mathrm{w}=\mathrm{a} / \mathrm{t}, \mathrm{h}=\mathrm{a} / \mathrm{c} / \mathrm{t}, \mathrm{m}=\mathrm{a} / \mathrm{c}, \mathrm{s}=\mathrm{g} / \mathrm{c}$.

\section{Acknowledgments}

The authors would like to sincerely thank Drs. Nina Jančar and Boštjan Mlakar for collecting clinical samples.

\section{Authors' contributions}

EMB performed the experiments, designed the study, analyzed the data and prepared drafts of the manuscript; LH designed the study, analyzed the data and prepared final version of the manuscript; DC and MFR analyzed the data and reviewed the final version of the manuscript; PEC performed the experiments and phylogenetic analysis; HB performed statistical analysis; KFK collected and processed samples and patient's data; MP and AAG analyzed the data and prepared final version of the manuscript. All authors read and approved the final manuscript.

\section{Funding}

This work was funded by: (i) Agencia Nacional de Promoción Científica y Tecnológica (Grant PICT-2012-0652); (ii) the Bilateral Cooperation Program between the Republic of Argentina and Republic of Slovenia entitled "Development of novel molecular methods for detection and identification of human papillomaviruses from genus Beta and Gamma and their comprehensive molecular-phylogenetic characterization in the oral cavity and nasopharynx" supported by the Ministerio de Ciencia, Tecnología e Innovación Productiva de Argentina (grant agreement no. AR/14/05) and the Slovenian Research Agency (grant agreement nos. P3-0083 and BI-AR/ 1517-005); and (iii) European Society of Clinical Microbiology and Infectious Diseases (ESCMID) Observership Program, granted to Elisa M. Bolatti. Elisa M. Bolatti and María F. Re-Louhau are supported by Post-Doctoral fellowships of CONICET. The funders had no role in the study design, data collection and analysis, nor in the preparation of the manuscript or the decision to publish.

\section{Availability of data and materials}

The datasets used and/or analyzed during the current study are available from the corresponding author on reasonable request.

\section{Ethics approval and consent to participate}

Swabs of the anal canal, cervical cancer biopsies, swabs and biopsies of genital warts, and oral swabs were obtained during our past or ongoing studies (Chouhy et al., 2010; Jančar et al., 2009; Milošević et al., 2010; Komloš et al., 2012; Mlakar et al., 2014; Hošnjak et al., 2015), in compliance with the Helsinki Declaration. Studies were approved by the National Ethics Committee of the Ministry of Health of Republic of Slovenia (consent nos. 131/06/07, 34/11/06, and 45/04/07) and by the Institutional Review Board of the Facultad de Ciencias Bioquímicas y Farmacéuticas, Universidad Nacional de Rosario, Rosario, Argentina (reference number 6060/134) and the Institutional Review Board of the Institute of Microbiology and Immunology, Faculty of Medicine, University of Ljubljana. Written informed consent was obtained from all patients, and only patient's gender, age and immune status were available to researchers.

\section{Consent for publication}

Not applicable.

\section{Competing interests}

The authors declare that they have no competing interests.

\section{Author details}

'Grupo Virología Humana, Instituto de Biología Molecular y Celular de Rosario (CONICET), Suipacha 590, 2000 Rosario, Argentina. ª́rea Virología, Facultad de Ciencias Bioquímicas y Farmacéuticas, Universidad Nacional de Rosario, Suipacha 531, 2000 Rosario, Argentina. ${ }^{3}$ Faculty of Medicine, Institute of Microbiology and Immunology, University of Ljubljana, Zaloška 4, 1000 Ljubljana, Slovenia. ${ }^{4}$ Área Estadística y Procesamiento de Datos, Facultad de 
Ciencias Bioquímicas y Farmacéuticas, Universidad Nacional de Rosario, Suipacha 531, 2000 Rosario, Argentina.

\section{Received: 20 February 2019 Accepted: 17 February 2020 Published online: 07 April 2020}

\section{References}

1. de Villiers EM, Fauquet C, Broker TR, Bernard HU, Zur HH. Classification of papillomaviruses. Virology. 2004;324(1):17-27.

2. de Villiers EM. Cross-roads in the classification of papillomaviruses. Virology. 2013:445(1-2):210

3. Van Doorslaer K, Li Z, Xirasagar S, Maes P, Kaminsky D, Liou D, et al. The papillomavirus episteme: a major update to the papillomavirus sequence database. Nucleic Acids Res. 2017;45(D1):499-506.

4. WHO/ICO Information Centre on HPV and Cervical Cancer (HPV Information Centre). Human Papillomavirus and Related Cancers in World. Summary Report. 2010.

5. Donà MG, Gheit T, Latini A, Benevolo M, Torres M, Smelov V, et al. Alpha, beta and gamma human papillomaviruses in the anal canal of HIV infected and uninfectedmen who have sex with men. J Inf Secur. 2015;71(1):74-84.

6. Combes JD, Heard I, Poizot-Martin I, Canestri A, Lion A, Piroth L, et al. ANRS EP57 APACHES study group. J Infect Dis. 2018;217(10):1535-43.

7. Frisch M, Biggar RJ, Goedert JJ. Human papillomavirus-associated cancers in patients with human immunodeficiency virus infection and acquired immunodeficiency syndrome. J Natl Cancer Inst. 2000;92(18):1500-10.

8. Yanik EL, Katki HA, Engels EA. Cancer risk among the HIV-infected elderly in the United States. AIDS. 2016;30(10):1663-8.

9. Nyitray AG. The epidemiology of anal human papillomavirus infection among women and men having sex with women. Sex Health. 2012;9(6): 538-46.

10. Sichero L, Nyitray AG, Nunes EM, Nepal B, Ferreira S, Sobrinho JS, HIM Study Group, et al. Diversity of human papillomavirus in the anal canal of men: the HIM study. Clin Microbiol Infect. 2015;21(5):502-9.

11. Milošević M, Poljak M, Mlakar B. Anal HPV infection in Slovenian men who have sex with men. Cent Eur J Med. 2010:5(2):698-703.

12. Antonsson A, Forslund O, Ekberg H, Sterner G, Hansson BG. The ubiquity and impressive genomic diversity of human skin papillomaviruses suggest a commensalic nature of these viruses. J Virol. 2000;74(24):11636-41.

13. Chen Z, Schiffman M, Herrero R, DeSalle R, Burk RD. Human papillomavirus (HPV) types 101 and 103 isolated from cervicovaginal cells lack an E6 open reading frame (ORF) and are related to gamma-papillomaviruses. Virology. 2007;360(2):447-53.

14. Bottalico D, Chen Z, Kocjan BJ, Seme K, Poljak M, Burk RD. Characterization of human papillomavirus type 120: a novel betapapillomavirus with tropism for multiple anatomical niches. J Gen Virol. 2012;93(8):1774-9.

15. Forslund $\mathrm{O}$, Johansson H, Madsen KG, Kofoed K. The nasal mucosa contains a large spectrum of human papillomavirus types from the Betapapillomavirus and Gammapapillomavirus genera. J Infect Dis. 2013; 208(8):1335-41.

16. Quint KD, Genders RE, de Koning MN, Borgogna C, Gariglio M, Bouwes Bavinck JN, et al. Human Beta-papillomavirus infection and keratinocyte carcinomas. J Pathol. 2015;235(2):342-54.

17. Nobre R, Herraez-Hernandez E, Fei JW, Langbein L, Kaden S, Gröne HJ, et al. E7 Oncoprotein of novel human papillomavirus type 108 lacking the E6 gene induces dysplasia in Organotypic keratinocyte cultures. J Virol. 2009; 83(7):2907-16

18. Grace M, Munger K. Proteomic analysis of the gamma human papillomavirus type 197 E6 and E7 associated cellular proteins. Virology. 2017;500:71-81.

19. Meyers JM, Grace M, Uberoi A, Lambert PF, Munger K. Inhibition of TGF- $\beta$ and NOTCH signaling by cutaneous papillomaviruses. Front Microbiol. 2018; 9:389.

20. Arroyo Mühr LS, Hultin E, Bzhalava D, Eklund C, Lagheden C, Ekström J, et al. Human papillomavirus type 197 is commonly present in skin tumors. Int J Cancer. 2015:136(11):2546-55.

21. Bolatti EM, Hošnjak L, Chouhy D, Re-Louhau MF, Casal PE, Bottai H, et al. High prevalence of Gammapapillomaviruses (Gamma-PVs) in pre-malignant cutaneous lesions of immunocompetent individuals using a novel broadspectrum primer system, and identification of HPV210, a novel Gamma-PV type. Virology. 2018;525:182-91.
22. Pastrana DV, Peretti A, Welch NL, Borgogna C, Olivero C, Badolato R, et al. Metagenomic Discovery of 83 New Human Papillomavirus Types in Patients with Immunodeficiency. mSphere. 2018;3(6):e00645-18.

23. Johansson H, Bzhalava D, Ekstrom J, Hultin E, Dillner J, Forslund O. Metagenomic sequencing of "HPV-negative"condylomas detects novel putative HPV types. Virology. 2013;44(1):01-7.

24. Arroyo Mühr LS, Bzhalava D, Lagheden C, Eklund C, Johansson H, Forslund $\mathrm{O}$, et al. Does human papillomavirus-negative condylomata exist? Virology. 2015:485:283-8.

25. Ekström J, Forslund O, Dillner J. Three novel papillomaviruses (HPV109, HPV112 and HPV114) and their presence in cutaneous and mucosal samples. Virology. 2010;397(2):331-6.

26. Ameur A, Meiring TL, Bunikis I, Haggqvist S, Lindau C, Lindberg JH, et al. Comprehensive profiling of the vaginal microbiome in HIV positive women using massive parallel semiconductor sequencing. Sci Rep. 2014:4:4398.

27. Mlakar B, Kocjan BJ, Hošnjak L, Fujs Komloš K, Milošević M, Poljak M. Betapapillomaviruses in the anal canal of HIV positive and HIV negative men who have sex with men. J Clin Virol. 2014;61(2):237-41.

28. Smelov V, Hanisch R, McKay-Chopin S, Sokolova O, Eklund C, Komyakov B, et al. Prevalence of cutaneous Beta and Gamma human papillomaviruses in the Anal Canal of men who have sex with women. Papillomavirus Res. 2017;3:66-72.

29. Jančar N, Kocjan BJ, Poljak M, Lunar MM, Bokal EV. Distribution of human papillomavirus genotypes in women with cervical cancer in Slovenia. Eur J Obstet Gynecol Reprod Biol. 2009;145(2):184-8.

30. Chouhy D, Gorosito M, Sanchez A, Serra EC, Bergero A, Fernandez Bussy R, et al. New generic primer system targeting mucosal/genital and cutaneous human papillomaviruses leads to the characterization of HPV 115, a novel Beta-papillomavirus species 3. Virology. 2010;397(1):205-16.

31. Komloš KF, Kocjan BJ, Košorok P, Luzar B, Meglič L, Potočnik M, et al. Tumorspecific and gender-specific pre-vaccination distribution of human papillomavirus types 6 and 11 in anogenital warts and laryngeal papillomas: a study on 574 tissue specimens. J Med Virol. 2012;84(8):1233-41.

32. Hošnjak L, Kocjan BJ, Pirš B, Seme K, Poljak M. Characterization of two novel Gammapapillomaviruses, HPV179 and HPV184, isolated from common warts of a renal-transplant recipient. PLoS One. 2015;10(3):e0119154.

33. Saiki RK, Bugawan TL, Horn GT, Mullis KB, Erlich HA. Analysis of enzymatically amplified beta-globin and HLA-DQ alpha DNA with allelespecific oligonucleotide probes. Nature. 1986;324(6093):163-6.

34. Tamura K, Stecher G, Peterson D, Filipski A, Kumar S. MEGA6: molecular evolutionary genetics analysis version 6.0. Mol Biol Evol. 2013;30(12):2725-9.

35. Katoh K, Rozewicki J, Yamada KD. MAFFT online service: multiple sequence alignment, interactive sequence choice and visualization. Brief Bioinform. 2017:20:1-7. https://doi.org/10.1093/bib/bbx108.

36. Drummond AJ, Suchard MA, Xie D, Rambaut A. Bayesian phylogenetics with BEAUti and the BEAST 1.7. Mol Biol Evol. 2012;29(8):1969-73.

37. Forman D, de Martel C, Lacey CJ, Soerjomataram I, Lortet-Tieulent J, Bruni L, et al. Global burden of human papillomavirus and related diseases. Vaccine. 2012;30(Suppl 5):F12-23.

38. Cubie HA. Diseases associated with human papillomavirus infection. Virology. 2013;445(1-2):21-34.

39. Iftner A, Klug SJ, Garbe C, Blum A, Stancu A, Wilczynski SP, Iftner T. The prevalence of human papillomavirus genotypes in nonmelanoma skin cancers of nonimmunosuppressed individuals identifies high-risk genital types as possible risk factors. Cancer Res. 2003;63(21):7515-9.

40. Latsuzbaia A, Arbyn M, Dutta S, Fischer M, Gheit T, Tapp J, et al. Complete Genome Sequence of a Novel Human Gammapapillomavirus Isolated from a Cervical Swab in Luxembourg. Genome Announc. 2018;6:e00114-8. https://doi.org/10.1128/genomeA.00114-18.

41. Smelov V, Muwonge R, Sokolova O, McKay-Chopin S, Eklund C, Komyakov B, et al. Beta and gamma human papillomaviruses in anal and genital sites among men: prevalence and determinants. Sci Rep. 2018;8(1):8241.

42. Murahwa AT, Meiring TL, Mbulawa ZZA, Williamson AL. Discovery, characterisation and genomic variation of six novel Gammapapillomavirus types from penile swabs in South Africa. Papillomavirus Res. 2019;7:102-11.

43. Torres M, Gheit T, McKay-Chopin S, Rodríguez C, Romero JD, Filotico R, Doná MG, Ortiz M, Tommasino M. Prevalence of beta and gamma human papillomaviruses in the anal canal of men who have sex with men is influenced by HIV status. J Clin Virol. 2015;67:47-51.

44. Van Doorslaer K, McBride AA. Molecular archeological evidence in support of the repeated loss of a papillomavirus gene. Sci Rep. 2016;6:33028. 
45. Ure AE, Forslund O. Characterization of human papillomavirus type 154 and tissue tropism of Gammapapillomaviruses. PLoS One. 2014;9(2):e89342.

46. Oštrbenk A, Kocjan BJ, Hošnjak L, Li J, Deng $Q$, Šterbenc A, et al. Identification of a novel human papillomavirus, type HPV199, isolated from a nasopharynx and anal canal, and complete genomic characterization of papillomavirus species Gamma-12. PLoS One. 2015;10(9):e0138628.

47. Chouhy D, D'Andrea RM, Iglesias M, Messina A, Ivancovich JJ, Cerda B, Galimberti D, Bottai H, Giri AA. Prevalence of human papillomavirus infection in Argentinean women attending two different hospitals prior to the implementation of the National Vaccination Program. J Med Virol. 2013; 85(4):655-66.

48. Moscicki AB, Ma Y, Gheit T, McKay-Chopin S, Farhat S, Widdice LE, et al. Prevalence and Transmission of Beta and Gamma Human Papillomavirus in Heterosexual Couples. Open Forum Infect Dis. 2017:4:0fw216.

49. Nyitray AG, Carvalho da Silva RJ, Baggio ML, Smith D, Abrahamsen M, Papenfuss $\mathrm{M}$, et al. Six-month incidence, persistence, and factors associated with persistence of anal human papillomavirus in men: the HPV in men study. J Infect Dis. 2011b;204(11):1711-22.

50. Sonnex C, Strauss S, Gray JJ. Detection of human papillomavirus DNA on the fingers of patients with genital warts. Sex Transm Infect. 1999;75(5):317-9.

51. Partridge JM, Hughes JP, Feng Q, Winer RL, Weaver BA, Xi LF, et al. Genital human papillomavirus infection in men: incidence and risk factors in a cohort of university students. J Infect Dis. 2007;196(8):1128-36.

52. Nyitray AG, Carvalho da Silva RJ, Baggio ML, Lu B, Smith D, Abrahamsen M, et al. Age-specific prevalence of and risk factors for anal human papillomavirus (HPV) among men who have sex with women and men who have sex with men: the HPV in men (HIM) study. J Infect Dis. 2011a; 203(1):49-57.

53. Bolatti EM, Chouhy D, Hošnjak L, Casal PE, Kocjan BJ, Bottai H, et al. Natural history of human papillomavirus infection of sunexposed healthy skin of immunocompetent individuals over three climatic seasons and identification of HPV209, a novel betapapillomavirus. J Gen Virol. 2017;98(6): 1334-48.

\section{Publisher's Note}

Springer Nature remains neutral with regard to jurisdictional claims in published maps and institutional affiliations.

Ready to submit your research? Choose BMC and benefit from:

- fast, convenient online submission

- thorough peer review by experienced researchers in your field

- rapid publication on acceptance

- support for research data, including large and complex data types

- gold Open Access which fosters wider collaboration and increased citations

- maximum visibility for your research: over $100 \mathrm{M}$ website views per year

At $\mathrm{BMC}$, research is always in progress.

Learn more biomedcentral.com/submissions 